Pacific Journal of Mathematic 


\section{$S(\alpha)$ SPACES AND REGULAR HAUSDORFF EXTENSIONS}

\section{JACK R. Porter ANd Charles VotaW}

A class of separation axioms $S(\alpha)$, one for each ordinal $\alpha>0$, is introduced. Axiom $S(1)$ is the Hausdorff property, $S(2)$ is Urysohn and regular implies $S\left(w_{0}\right)$, where $w_{0}$ is the first infinite ordinal. Minimal $S(\alpha)$ and $S(\alpha)$-closed spaces are characterized, and many of the known results for minimal $S(i)$ and $S(i)$-closed are extended, $i=1,2$. For a limit ordinal $\alpha>0$, minimal $S(\alpha)$ spaces are shown to be regular. A new approach to the study of minimal regular spaces is provided by showing that the properties of minimal regular and minimal $S\left(w_{0}\right)$ are equivalent even though the concepts of regularity and $S\left(w_{0}\right)$ are not equivalent.

A new subclass of regular spaces-called $O C E$-regular spaces-is introduced and used to develop an extension theory for regular spaces, which subsumes the regular-closed extension theory developed by Harris. It is proven that every regular space can be densely embedded in an $O C E$-regular space and that the set of $O C E$-regular extensions of a regular space is in a one-to-one correspondence with a set of generalized Smirnov proximities compatible with the regular space.

1. Introduction. It is well-known that the properties of regularity (includes $T_{1}$ ) and complete Hausdorff (every pair of distinct points can be separated by a real-valued continuous function) are independent of each other and yet, are implied by completely regularity (includes $T_{1}$ ) and imply Urysohn (every pair of distinct points can be separated by closed neighborhoods). In $\$ 2$ a method of distinguishing between regular and completely Hausdorff is developed by defining a class of separation axioms $S(\alpha)$, one for each ordinal $\alpha>0$. This class can be thought of as a measuring rod since it is linearly ordered in the sense that an $S(\beta)$ space is $S(\alpha)$ if $\beta \geqq \alpha$. In particular, if $w_{0}$ denotes the first infinite ordinal and $w_{1}$ the first uncountable ordinal, we prove that a regular space is $S\left(w_{0}\right)$ but not necessarily $S\left(w_{0}+1\right)$ whereas a completely Hausdorff space is $S(\alpha)$ for any ordinal $\alpha<w_{1}$ but not necessarily $S\left(w_{1}\right)$.

In $\S 3$ we study and characterize minimal $S(\alpha)$ and $S(\alpha)$-closed spaces. Known results about minimal topological spaces, cf. [8], are extended, and a new approach to minimal regular spaces is provided. Surprisingly, for a limit ordinal $\alpha>0$, a minimal $S(\alpha)$ space is regular, and a space is minimal regular if and only if it is minimal $S\left(w_{0}\right)$ even though the concepts of regularity and $S\left(w_{0}\right)$ are not equivalent. A space is shown to be regular-closed if and only if the space is regular 
and $S\left(w_{0}\right)$-closed. $S\left(w_{0}\right)$ spaces are shown to be densely embeddable in $S\left(w_{0}\right)$-closed spaces even though the corresponding fact for regular spaces is false. We conclude the section by proving that a space that is the countable union of nowhere dense, compact sets is not Katětov- $S(n)$ for any finite ordinal $n>0$; this theorem, for $n=1$, was recently proved, independently, by Mioduszewski [21].

The extension theories for regular spaces, like the extension theories for completely regular spaces, are either completion theories [20] or topological theories [14]. One topological theory (the only one as far as we know) is that of regular-closed extensions. Harris characterizes the regular-closed extensions of a regular space by first generalizing Smirnov's proximities for completely regular spaces to a proximity (called $R$-proximity) compatible with the topology of a regular space. One of the liabilities of the regular-closed extension theory for regular spaces, as observed by Harris, is that some regular spaces have no regular-closed extensions. In $\S 4$, we prove that any topological extension theory without this defect must have one of the assets of the regular-closed extension theory as a liability. We develop a topological extension theory for regular spaces which bypasses the defect of the regular-closed extension theory by using a mixture of $R$-proximities of Harris [14] and a completion method by Leader [20].

A subset $A$ of a space $(X, \tau)$ is regular-open if $A=\operatorname{int}(\mathrm{cl} A)$; the set of regular-open sets form a basis for a topology [10, p. 138] denoted as $\tau_{s} ; \tau_{s}$ is called the semiregularization of $\tau$. A space $(X, \tau)$ is semiregular if $\tau=\tau_{s}$, in particular, $\left(X, \tau_{s}\right)$ is semiregular.

An extension of $X$ is a space $Y$ in which $X$ is a dense subspace; for a topological property $P$, an extension $Y$ of $X$ is a $P$-extension if $Y$ possesses property $P$. In particular, a regular extension $Y$ of $X$ is a regular (includes Hausdorff) space $Y$ which is an extension of $X$. Let $Z$ and $Y$ be extensions of $X ; Z$ is projectively larger than (resp. injectively larger than, isomorphic to) $Y$ if there is a continuous surjection $f: Z \rightarrow Y$ (resp. embedding $f: Y \rightarrow Z$, homeomorphism $f: Z \rightarrow$ $Y$ ) that leaves $X$ pointwise fixed, i.e., $f(x)=x$ for $x \in X$. Let $\mathscr{E}$ be a class of extensions of a space $X$. Then "isomorphism" is an equivalence relation on $\mathscr{E}$, and we consider isomorphic extensions as the same extension. Also, "projectively larger" and "injectively larger" are preorders on $\mathscr{E}$, and the terms projective maximums, injective maximums, projective maximals, and injective maximals refer to maximum and maximal elements relative to these preorders on $\mathscr{E}$.

Let $P$ be a topological property. A space $X$ with property $P$ is called $P$-closed (resp. minimal $P$ ) if $X$ is a closed subspace in every $P$-space in which it is embedded (resp. has no strictly coarser $P$ topologies). A space is Katétov- $P$ if it has a coarser minimal $P$ 
topology. Much of the known results about $P$-closed, minimal $P$, and Katètov- $P$ spaces are presented in [8].

A filter on a space $X$ is called open if it is generated by a filter base of open sets. A regular filter on $X$ is an open filter that is generated by a filter base of closed sets. A completely regular filter $\mathscr{F}$ on $X$ is an open filter with the property that for each $U \in \mathscr{F}$, there is $V \in \mathscr{F}$ and a real-valued continuous function $f$ such that $f(V)=$ $\{0\}$ and $f(X \backslash U) \subseteq\{1\}$. The adherence of a filter $\mathscr{F}$ on a space $X$ is $\cap\{\mathrm{cl} F: F \in \mathscr{F}\}$ and denoted as $a(\mathscr{F})$. A filter with void adherence is called free, otherwise it is called fixed. The neighborhood filter of a point $x$ in a space is denoted as $\mathscr{N}_{x}$.

The symbols $\boldsymbol{N}, \boldsymbol{Z}$, and $\boldsymbol{R}$ are used to denote, respectively, the set of positive integers, and real numbers.

2. Basic properties of $S(\alpha)$. Two filters $\mathscr{F}$ and $\mathscr{G}$ on a space $X$ are called $R(\alpha)$-separated (respectively, $U(\alpha)$-separated) for an ordinal $\alpha>0$ if there are open subfamilies $\left\{F_{\beta}: \beta<\alpha\right\} \subseteq \mathscr{F}$ and $\left\{G_{\beta}: \beta<\alpha\right\} \subseteq \mathscr{G}$ such that $F_{0} \cap G_{0}=\varnothing$ (resp. cl $\left.F_{0} \cap \operatorname{cl} G_{0}=\varnothing\right\}$ and for $\gamma+1<\alpha, \mathrm{cl} G_{\gamma+1} \subseteq G_{\gamma}$ and $\mathrm{cl} F_{\gamma+1} \subseteq F_{\gamma}$; sometimes, we say that the open subfamilies are $R(\alpha)$-separated (resp. $U(\alpha)$-separated). Corresponding to the definitions in $[P]$, a space $X$ is said to be $R(\alpha)$ (respectively, $U(\alpha)$ ) if for every pair of distinct points $x, y \in X, \mathscr{N}_{x}$ and $\mathscr{N}_{y}$ are $R(\alpha)$-separated (resp. $U(\alpha)$-separated). For $\alpha \geqq w_{0}$, it is easy to verify that $R(\alpha)$ and $U(\alpha)$ are equivalent concepts; so, for notational convenience, the symbols $R(\alpha)$ and $U(\alpha)$ for $\alpha \geqq w_{0}$ are replaced by a single symbol $S(\alpha)$ and for $n \in N, R(n)$ is replaced by $S(2 n-1)$ and $U(n)$ is replaced by $S(2 n)$. In particular, for $n \in N$, our separation axiom $S(n)$ corresponds to $\bar{T}_{n}$ defined in [V].

The following facts are straightforward to prove and left to the reader.

(2.1) For each ordinal $\alpha>0$, an $S(\alpha+1)$ space is $S(\alpha)$.

(2.2) A space is Hausdorff if and only if it is $S(1)$ and is Urysohn if and only if it is $S(2)$.

(2.3) A regular Hausdorff space is $S\left(w_{0}\right)$ and a completely Hausdorff space is $S(\alpha)$ for any ordinal $\alpha<w_{1}$.

(2.4) A space is $S(\alpha)$ for each ordinal $\alpha>0$ if and only if the quasicomponents are singletons. In particular, such a space is completely Hausdorff.

(2.5) A subspace of an $S(\alpha)$ space is $S(\alpha)$.

(2.6) A product of nonvoid spaces is $S(\alpha)$ if and only if each 
coordinate space is $S(\alpha)$.

(2.7) The semiregularization of an $S(\alpha)$ space is also $S(\alpha)$.

(2.8) $S(\alpha)$ is an expansive property, i.e., a topology finer than an $S(\alpha)$ topology is also $S(\alpha)$.

The results of (2.3) are the best possible as demonstrated by the next theorem and examples. Recall that a space satisfies the $\aleph$-Suslin condition, for a cardinal $\boldsymbol{\aleph}$, whenever every family of pairwise disjoint, nonempty open sets has cardinality $\leqq \aleph$. For a cardinal $\aleph_{\beta}$, we let $w_{\beta}$ denote the least ordinal with cardinality $\aleph_{\beta}$.

Theorem 2.9. Let $X$ be a connected, $S(\alpha)$ space with more than one point. If $X$ satisfies the $\aleph_{\beta}$ Suslin condition, then $\alpha<w_{\beta+1}$.

Proof. Let $x$ and $y$ be distinct points. There is an open subfamily $\left\{U_{\gamma}: \gamma<\alpha\right\} \subseteq \mathscr{N}_{x}$ such that $y \notin U_{0}$ and $\operatorname{cl} U_{\gamma+1} \subseteq U_{\gamma}$ for $\gamma+1<$ $\alpha$. By $\boldsymbol{\aleph}_{\beta}$-Suslin condition, $\left\{U_{\gamma} \mid \operatorname{cl} U_{\gamma+1}: \gamma+1<\alpha\right\}$ has cardinality $\leqq \boldsymbol{\aleph}_{\beta}$; so, $\alpha<w_{\beta+1}$.

It is trivial to verify that a space with weight $\leqq \aleph$ or with a dense subset of cardinality $\leqq \aleph$ satisfies the $\aleph$-Suslin condition. Thus, it follows from Theorem 2.9 that the space of real numbers with the usual topology is $S(\alpha)$ for $\alpha<w_{1}$ but is not $S\left(w_{1}\right)$.

We now give an example of a regular space that is not $S\left(w_{0}+1\right)$ and an example of a $S(\alpha)$ space that is not $S(\alpha+1)$.

EXAmple 2.10. Let $\alpha, \beta$ be ordinals and $T_{r}$, for $-\alpha<\gamma<\beta$, be a copy of the deleted Tychonoff plank (i.e., $T_{\gamma}=\left(A_{1} \times A_{2} \backslash\left\{\left(w_{0}, w_{1}\right)\right\}\right) \times$ $\{\gamma\}$ where $A_{i}=$ ordinals $\left.\mu: 1 \leqq \mu \leqq w_{i-1}\right\}$ with the order topology, for $i=1,2)$. Let $X_{\alpha, \beta}$ be the quotient space of the topological sum of $\left\{T_{\gamma}:-\alpha<\gamma<\beta\right\}$ with $\left(n, w_{1}, \gamma\right)$ identified to $\left(n, w_{1}, \gamma+1\right)$ for $n<w_{0}$ and $\gamma$ even (limit ordinals are even) and with $\left(w_{0}, \delta, \gamma\right)$ identified to $\left(w_{0}, \delta, \gamma+1\right)$ for $\delta<w_{1}$ and $\gamma$ odd. Consider the coarser topology on $X_{\alpha, \beta}$ by enlarging the neighborhoods at the points $(m, \delta, \gamma)$ of $T_{\gamma}$, where $\gamma$ is a limit ordinal, to include some $\{(p, \varepsilon, \nu): n<p \leqq m, \eta<$ $\varepsilon \leqq \delta, \xi<\nu \leqq \gamma\}$ for some $n<m, \eta<\delta$, and $\xi<\gamma$. Similarly, the neighborhoods at the points $T_{-r}$, where $\gamma$ is a limit ordinal, are enlarged. Let $Y_{\alpha, \beta}$ denote the set $X_{\alpha, \beta}$ plus two additional points $b$ and $a$. When both $\alpha$ and $\beta$ are not the immediate successor of limit ordinals, a topology $\sigma$ is defined on $Y_{\alpha, \beta}$ by $U \in \sigma$ if $U \cap X_{\alpha, \beta}$ is open in $X_{\alpha, \beta}$ and $b \in U$ (respectively, $a \in U$ ) implies

(i) $T_{\gamma} \subseteq U\left(\operatorname{resp} . T_{-r} \subseteq U\right.$ ) for $\gamma>$ some $\xi$ if $\beta$ (resp. $\alpha$ ) is a limit ordinal,

(ii) $\left\{(p, \varepsilon, \beta-1) \in T_{\beta-1}: n<p<w_{0}, \eta<\varepsilon<w_{1}\right\} \subseteq U$ for some $n<$ $w_{0}$ and $\eta<w_{1}$ if $\beta$ is a nonlimit ordinal, and 
(iii) $\left\{(p, \varepsilon,-\alpha+1) \in T_{-\alpha+1}: n<p<w_{0}, \eta<\varepsilon<w_{1}\right\} \subseteq U$ for some $n<w_{0}$ and $\eta<w_{1}$ if $\alpha$ is a nonlimit ordinal.

(a) If $\alpha=\beta=w_{0}$, then $Y_{\alpha, \beta}$ is the well-known deleted Tychonoff spiral and such a space is regular [7] but not $S\left(w_{0}+1\right)$.

(b) If $k$ is a positive integer, then $Y_{k, k+1}$ is an $S(2 k-1)$ space which is not $S(2 k)$ and $Y_{k+1, k+1}$ is an $S(2 k)$ space which is not $S(2 k+1)$.

(c) If $\alpha$ is a limit ordinal, $Y_{\alpha, \alpha}$ is an $S(\alpha)$ space which is not $S(\alpha+1)$. (d) If $\alpha$ is an infinite nonlimit ordinal, $Y_{\alpha+1, \alpha+1}$ is an $S(\alpha)$ space which is not $S(\alpha+1)$.

3. Minimal $S(\alpha)$ spaces. An open filter $\mathscr{F}$ on a space $X$ is an $S(\alpha)$-filter if for $x \notin a(\mathscr{F}), \mathscr{F}$ and $\mathscr{N}_{x}$ are $S(\alpha)$-separated. The proofs of 3.1 and 3.2 are similar to the Hausdorff and Urysohn cases, cf. $[16,24]$.

(3.1) (a) An $S(\alpha)$ space is $S(\alpha)$-closed if and only if every $S(\alpha)$ filter is fixed.

(b) An $S(\alpha)$ space is minimal $S(\alpha)$ if and only if every $S(\alpha)$ filter with at most one adherent point is convergent.

(c) A minimal $S(\alpha)$ space is $S(\alpha)$-closed.

(d) A compact $S(\alpha)$ space is minimal $S(\alpha)$; in particular, a compact Hausdorff space is minimal $S(\alpha)$, for $\alpha<w_{1}$.

(e) $S(\alpha)$-closure is preserved by a continuous function onto an $S(\alpha)$ space.

(f) A minimal $S(\alpha)$ space is semiregular.

(3.2) Every $S(\alpha)$ space can be densely embedded in an $S(\alpha)$ closed space.

Theorem 3.3 (a) A minimal $S(\alpha)$ space, where $\alpha$ is a limit ordinal, is regular.

(b) An $S(\alpha+1)$, minimal $S(\alpha)$ space is regular.

Proof. The proof of (b) is similar to the proof of (a). To prove (a), let $X$ be a minimal $S(\alpha)$ space where $\alpha$ is a limit ordinal. Let $x \in X$ and $U$ be an open set containing $x$. For each $y \neq x$, there are $S(\alpha)$-separated open families $\left\{U_{\beta, y}: \beta<\alpha\right\} \subseteq \mathscr{N}_{y}$ and $\left\{V_{\beta, y}: \beta<\alpha\right\} \subseteq \mathscr{N}_{x}$. The filter $\mathscr{F}$ generated by $\left\{\bigcap_{T} V_{\beta, y}: T\right.$ is finite subset of $\left.\alpha \times(X \backslash\{x\})\right\}$ is :an $S(\alpha)$-filter converging to $x$. So, there is a finite subset $T \subseteq$ $\alpha \times(X \backslash\{x\})$ such that $\bigcap_{t} V_{\beta, y} \subseteq U$. Let $R=\{(\beta+1, y):(\beta, y \in T\}$. Since $\alpha$ is a limit ordinal, $V_{\gamma, y}$ is defined for $(\gamma, y) \in R$. Then $x \in$ $\bigcap_{R} V_{r, y}$ and $\operatorname{cl}\left(\bigcap_{R} V_{r, y}\right) \subseteq U$.

CoROLlary 3.4. A completely Hausdorff, minimal $S(\alpha)$ space for $\alpha \leqq w_{1}$ is regular. 
A point $p$ in a space $X$ is a regular point if for each open set $U$ containing $p$, there is an open set $V$ containing $p$ such that $\operatorname{cl} V \subseteq U$.

Lemma 3.5. Let $(X, \tau)$ be an $S\left(w_{0}\right)$ space and $x \in X$. There is a coarser $S\left(w_{0}\right)$ topology $\sigma$ on $X$ such that the regular points of $(X, \sigma)$ are the regular points of $(X, \tau)$ plus the point $x$.

Proof. Let $\mathscr{F}$ be the filter in the proof of Theorem 3.3 (a) where the limit ordinal is $w_{0}$. Define $\sigma$ by $W \in \sigma$ if $W \in \tau$ and $x \in W$ implies $W \in \mathscr{F}$, for $W \subseteq X$. $(X, \sigma)$ has the desired properties.

Lemma 3.6. Let $\mathscr{F}$ be an $S\left(w_{0}\right)$-filter on a space $X$. The set of all regular filters contained in $\mathscr{F}$ contains a maximum element (denoted as $\left.\mathscr{F}_{r}\right)$ when partially ordered by set inclusion, and $a\left(\mathscr{F}_{r}\right)=a(\mathscr{F})$.

Proof. Let $\mathscr{S}=\{\mathscr{C} \subseteq \mathscr{F}: \mathscr{Y}$ is a regular filter $\}$, and let $\mathscr{F}_{r}=\cup \mathscr{S}$.

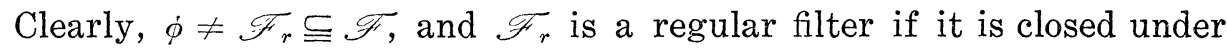
finite intersections. Let $U, V \in \mathscr{F}_{r}$. There are open families $\left\{U_{n}: n \in\right.$ $N\},\left\{V_{n}: n \in N\right\} \cong \mathscr{F}_{r}$ such that $U \supseteqq \operatorname{cl} U_{1}, V \supseteqq \operatorname{cl} V_{1}, U_{n} \supseteqq \operatorname{cl} U_{n+1}$ and $V_{n} \supseteqq \operatorname{cl} V_{n+1}$ for $n \in N$. The filter generated by $\{U \cap V\} \cup\left\{U_{n} \cap V_{n}: n \in\right.$ $N\}$ is a regular filter and an element of $\mathscr{S}_{\bullet}$ Thus, $U \cap V \in \mathscr{F}_{r}$. Since $\mathscr{F}_{r} \cong \mathscr{F}$, then $a(\mathscr{F}) \leqq a\left(\mathscr{F}_{r}\right)$. Suppose $p \notin a(\mathscr{F})$. Then $\mathscr{F}$ and $\mathscr{N}_{p}$ are $S\left(w_{0}\right)$-separated, and there is an open family $\left\{F_{n}: n \in N\right\} \subseteq$ $\mathscr{F}$ such that $p \notin \mathrm{cl} F_{1}$ and $F_{n} \supseteqq \operatorname{cl} F_{n+1}$ for $n \in N$. The filter generated by $\left\{F_{n}: n \in N\right\}$ is a regular filter, and $p \notin a\left(\mathscr{F}_{r}\right)$.

THEOREM 3.7. (a) A space is regular-closed if and only if it is $S\left(w_{0}\right)$-closed and regular.

(b) A space is minimal $S\left(w_{0}\right)$ if and only if it is minimal regular.

Proof. The "only if" part of (a) follows from Lemma 3.6 and 3.1(a). The "if" part of (a) follows from the fact that a regular filter on a regular space is an $S\left(w_{0}\right)$-filter. The "only if" part of (b) follows from Theorem 3.3, 3.1(b), and the fact that a regular filter on a regular space is an $S\left(w_{0}\right)$-filter. To show the "if" part of (b), let $(X, \tau)$ be a minimal regular space and let $\sigma$ be a coarser $S\left(w_{0}\right)$ topology on $X$. By Lemma 3.5, for each point $x \in X$, there is a coarser $S\left(w_{0}\right)$ topology $\rho$ on $X$ such that $\mathscr{N}_{x, \rho}\left(\mathscr{N}_{x}\right.$ relative to $\left.\rho\right)$ is a regular filter on $(X, \rho)$. Since $\rho \leqq \sigma \leqq \tau$, then $\mathscr{N}_{x, \rho}^{*}$ is a regular filter on $(X, \tau)$ and $x$ is the unique $\tau$-adherent point. By a theorem in $[3,7], \mathscr{N}_{x, o}$ converges to $x$ in $(X, \tau)$. Since this is true for each $x \in X$, then $\tau=\sigma$.

Corollary 3.8. A space is Katètov-S(wo $w_{0}$ if and only if it is Katětov-regular. 
A useful characterization of $S(n)$-closed spaces, for $n \in N$, is in terms of covers. Recall that a cover $\mathscr{A}$ is a shrinkable refinement of a cover $\mathscr{B}$ if for each $A \in \mathscr{A}, \mathrm{cl} A \subseteq B$ for some $B \in \mathscr{B}$. An open cover $\mathscr{U}$ of a space has an $n$-chain of shrinkable refinements if there are open covers $\mathscr{U}_{C}=\mathscr{U}_{0}, \mathscr{U}_{1}, \cdots, \mathscr{U}_{n}$ such that $\mathscr{U}_{i+1}$ is a shrinkable refinement of $\mathscr{U}_{i}$ for $i=0,1, \cdots, n-1$.

(3.9) (a) An $S(n)$ space is $S(n)$-closed if and only if every open cover with an $n-1$ chain of shrinkable refinements has a finite subfamily whose closures form a cover.

(b) An $S(n)$-closed space is compact if and only if it is regular.

(c) A minimal $S(n)$ space is compact if and only if it is $S(n+1)$.

Proof. The proof of (a) is similar to the Urysohn case [16], (b) follows immediately from (a), and (c) follows immediately from (b) and Theorem 3.3(b).

The results of 3.9 extend theorems in $[2,16,18,9,23]$. Our next theorem (the Hausdorff case was independently obtained by Mioduszewski [21]) enables us to extend solutions of previous problems in the Hausdorff and Urysohn cases.

THEOREM 3.10. A space which is the countable union of nowhere dense, compact subsets is not $S(k)$-closed for any $k \in N$.

Proof. Let $X=\cup\left\{A_{n}: n \in N\right\}$, where $A_{n}$ is a nowhere dense, compact subset. Assume $X$ is $S(k)$-closed for some $k \in N$. For $n \in N$, let $B_{n}=A_{1} \cup \cdots \cup A_{n}$. Each $B_{n}$ is a nowhere dense, compact set, and since each $B_{n} \neq X$, then $\left\{B_{n}: n \in N\right\}$ is an infinite set. By renumbering if necessary, we can assume that $B_{n} \varsubsetneqq B_{n+1}$ for $n \in N$. Let $p_{1} \in B_{2} \backslash B_{1}$. Using the compactness of $B_{1}$, it follows that $\mathscr{N}_{p_{1}}$ and $\mathscr{N}_{B_{1}}$ (the set of all neighborhoods of $B_{1}$ ) are $S(k)$-separated. So, there are $S(k)$-separated open families $\left\{V_{1, i}: 0 \leqq i<k\right\} \subseteq \mathscr{N}_{B_{1}}$ and $\left\{U_{1, i}: 0 \leqq i<k\right\} \cong$ $\mathscr{N}_{p_{1}}$. Since $B_{2}$ is nowhere dense, then $U_{1, k-1} \nsubseteq B_{2}$. Let $t$ be the first integer greater than 1 such that $U_{1, k-1} \cap\left(B_{t+1} \mid B_{t}\right) \neq \varnothing$, and let $p_{2} \in$ $U_{1, k-1} \cap B_{t+1} \mid B_{t}$. There are $S(k)$-separated open families $\left\{V_{2, i}: 0 \leqq i<k\right\} \subseteq$ $\mathscr{N}_{B_{t}}$ and $\left\{U_{2, i}: 0 \leqq i<k\right\} \subseteq \mathscr{N}_{p_{2}} \cdot$ By induction, we obtain a family of open sets $\left\{U_{n, i}: 0 \leqq i<k, n \in N\right\}$ with the finite intersection property. The filter generated by $\left\{U_{n, k-1}: n \in N\right\}$ is a free $S(k)$-filter, a contradiction.

COROLLARY 3.11. A space that is the countable union of nowhere dense compact sets is not Katětov-S(n), for all $n \in N$.

Proof. If $X$ is a Katétov- $S(n)$ space that is the countable union of nowhere dense compact sets, then $X$ has a coarser minimal $S(n)$ 
topology which is $S(n)$-closed by 3.1(c). Since nowhere dense, compact sets remain nowhere dense and compact in coarser Hausdorff topologies, then $X$ with the coarser $S(n)$-closed topology is the countable union of nowhere dense, compact sets-a contradiction to Theorem 3.10 .

An immediate consequence of Corollary 3.11 is that a countable space without isolated points is not Katétov- $S(n)$, in particular, the space of rational numbers. In response to a problem by Bourbaki [9], Herrlich [16] shows that the space of rational numbers is not KatětovHausdorff; Herrlich also proves that the space of rational numbers is not Katětov-regular. In light of Theorem 3.7, we can conclude that the space of rational numbers is not Katětov-S( $\alpha)$ for $\alpha \leqq w_{0}$.

CoRollary 3.12. If the set $A$ of nonisolated points of an $S(k)$ closed space is countable, then $A$ is nowhere dense.

Proof. Let $X$ be $S(k)$-closed and $A=\left\{x_{n}: n \in N\right\}$. Suppose $U \subseteq$ $A$ is a nonempty open set. Let $U \cap A=\left\{x_{n(i)}: i \in N\right\}$, and let $B_{m}=$ $\left\{x_{i}: 1 \leqq i \leqq n(m)\right\}$. Proceed as in proof of Theorem 3.10 where $p_{1}=$ $x_{n(2)}, t$ is the first integer greater than 1 such that $U \cap U_{1, k \rightarrow 1} \cap$ $\left(B_{t+1} \mid B_{t}\right) \neq \varnothing$, and $p_{2} \in U \cap U_{1, k-1} \cap\left(B_{t+1} \mid B_{t}\right)$. As in the proof of Theorem 3.10, a free $S(k)$-filter is obtained which is a contradiction.

The Hausdorff case of Corollary 3.12 is an exercise in [10], and the Urysohn case is a lemma in [26]. Using Corollary 3.12 and the same argument as for the Urysohn case [26], the next corollary is easily obtained.

CoROLlaRY 3.13. A countable, minimal $S(n)$ space is compact for $n>1$.

The space in Example 2.10(a) is a noncompact, minimal $S\left(w_{0}\right)$ space, and the semiregularizations of the spaces in Example 2.10(b) are noncompact, minimal $S(k)$ spaces. The authors are unaware of a noncompact, minimal $S(\alpha)$ space fo $\alpha>w_{0}$ but conjecture that such exist. We now give examples (1) to show the converse of $3.1(\mathrm{c})$ is false, (2) of an $S\left(w_{0}\right)$ space with a regular filter that is not an $S\left(w_{0}\right)$ filter, and (3) of an $S\left(w_{0}\right)$-closed space that is not Katětov- $S\left(w_{0}\right)$.

\section{EXAMPLE 3.14 .}

(a) [29]. Let $X=\{a\} \cup\left\{b_{i j}\right\} \cup\left\{c_{i}\right\}$ where $i, j \in N$. A set $U \subseteq X$ is defined to be open if $a \in U$ implies $\left\{b_{i j}: j \in N\right.$ and $\left.i \geqq n\right\} \subseteq U$ for some $n \in N$ and $c_{i} \in U$ implies $\left\{b_{i j}: j \geqq n\right\} \subseteq U$ for some $n \in N . \quad X$ is $S(\alpha)$-closed for all ordinals $\alpha>0$ but is not minimal $S(\alpha)$.

(b) [25]. Let $\tau$ denote the usual topology on unit interval $I$. 
Partition $I$ into subsets $X(n)$, for $n \in N$; such that each $X(n)$ is dense in $I$. Let $\sigma$ be the topology on $I$ generated by $\tau \cup\{X(2 n-1): n \in N\} \cup$ $\{X(2 n-1) \cup X(2 n) \cup X(2 n+1): n \in N\}$. Stephenson [25] proved that $(I, \sigma)$ is completely Hausdorff-closed and contains a free regular filter generated by $\{F(i, n): i, n \in N\}$ where $F(i, n)=(x-1 / i, x+1 / i) \cap$ $\cup\{X(j): j \geqq 2 n-1\}$ and $x \in X(1)$. It is straightforward to show that $(I, \sigma)$ is $S(\alpha)$-closed for $w_{0}<\alpha<w_{1} . \quad(I, \sigma)$ is not $S\left(w_{1}\right)$-closed since it is not $S\left(w_{1}\right)$ by Theorem 2.9. The free regular filter defined by Stephenson is not an $S\left(w_{0}\right)$-filter. For $k \in N$, let $G_{k}=\cup\{(1 / n, 1 /(k+2)) \cap$ $(X(2 n+4 k-4) \cup X(2 n+4 k-5)): n \geqq k+3\} ; G_{k}$ is open since for $n \geqq k+3, \quad(1 / n, 1 /(k+2)) \cap X(2 n+4 k-5)$ and $(1 / n, 1 /(k+2)) \cap$ $(X(2 n+4 k-5) \cup X(2 n+4 k-4) \cup X(2 n+4 k-3))$ are open. It easily follows that $\left\{G_{k} ; k \in N\right\}$ is a free $S\left(w_{0}\right)$-filter base implying that $(I, \sigma)$ is not $S\left(w_{0}\right)$-closed.

(c) Partition $I \backslash\{0\}$ into sets $X(n)$, for $n \in Z$, such that $X(n)$ is dense in $I$ and $(1 / n: n \in N\} \subseteq X(0)$. For $n \in Z \backslash\{0\}$, let $Y(n)=$ $(0,1 /(|n|+1)) \cap X(n))$, and let $Y(0)=I \backslash(\{0\} \cup \cup\{Y(n): n \in Z \backslash\{0\}\})$. Let $\tau$ be the usual topology on $I \backslash\{0\})$, and let $\sigma$ be the topology generated by $\tau \cup(Y(2 n): n \in Z\} \cup\{Y(2 n) \cup Y(2 n+1) \cup Y(2 n+2): n \in Z\}$. Let $Y$ denote $I \backslash\{0\}$ plus two additional points $a$ and $b$. A set $U \subseteq Y$ is defined as open if $U \cap I \in \sigma$ and $a \in U$ (respectively, $b \in U$ ) implies $Y(m) \subseteq U$ (resp. $Y(-m) \subseteq U$ ) for $m>n$ for some $n \in N$. The space $Y$ is $S\left(w_{0}\right)$-closed and has no coarser regular topology. By Theorem 3.7, $Y$ is not Katětov-S $\left(w_{0}\right)$.

4. An extension theory for regular spaces. Before we develop an extension theory for regular spaces, it is necessary to investigate theories in a general setting. Let $\mathscr{C}$ be a class of topological spaces closed under homeomorphic images. An element of $\mathscr{C}$ is $\mathscr{C}$-closed if it has no proper extension in $\mathscr{C}$. Using the usual extension theories [5] as a guide, an extension theory of $\mathscr{C}$ is a class $\mathscr{E}$ of spaces (closed under homeomorphic images) satisfying at least:

(E1) $\mathscr{E} \subseteq \mathscr{C}$.

(E2) Each element of $\mathscr{C}$ has an $\mathscr{E}$-extension, i.e., each element of $\mathscr{C}$ is densely embeddable in a space of $\mathscr{E}$.

The compact Hausdorff extension theory of completely regular spaces also satisfies:

(E3) An element of $\mathscr{E}$ has no proper $\mathscr{E}$-extension.

THEOREM 4.1. Let $\mathscr{C}$ and $\mathscr{C}$ be classes of spaces satisfying E1, E2 and E3. Then $\mathscr{E}$ is precisely the class of $\mathscr{C}$-closed elements of $\mathscr{C}$.

Proof. Suppose $X \in \mathscr{E}$, and assume $X$ is not $\mathscr{C}$-closed. Then $X$ 
has a proper $\mathscr{C}$-extension, say $Y$. By E2, $Y$ has an $\mathscr{E}$-extension which is a proper $\mathscr{E}$-extension of $X$, contradicting E3. To show the converse, let $X$ be a $\mathscr{C}$-closed space. By E2, $X$ has an $\mathscr{E}$-extension $Y$. Since $Y \in \mathscr{C}$ by E1, then $X$ and $Y$ are homeomorphic and $X \in \mathscr{E}$.

Since the minimal Hausdorff extensions of semiregular Hausdorff spaces satisfy E1, E2, and E3 [4], then the following result is immediate.

CoROLlary 4.2. [4] A space is semiregular Hausdorff-closed if and only if it is minimal Hausdorff.

It is well-known [3, 6] that compact Hausdorff spaces are precisely the completely regular-closed spaces. Since there are noncompact, realcompact spaces, then the realcompact extension theory of completely regular spaces does not satisfy E3 although E1 and E2 are satisfied. It follows from the definition of $\mathscr{C}$-closed, that the class of $\mathscr{C}$-closed spaces satisfy E1 and E3. The class of $\mathscr{C}$-closed spaces also satisfies E2 whenever $\mathscr{C}$ is the class of completely Hausdorff spaces [27] or the class of $S(\alpha)$ spaces by 3.2, but does not satisfy E2 when $\mathscr{C}$ is the class of regular spaces [16]. The regular-closed extension theory for regular spaces has been characterized in terms of proximities by Harris [14]. In the remainder of this paper, we advance an extension theory for regular spaces satisfying E1 and $\mathrm{E} 2$ and characterize the extension theory in terms of proximities.

The following useful result by Banaschewski [5] is frequently needed in the sequel.

THEOREM 4.3. [5]. Let $Y$ be a regular extension of $X$, and for each $y \in Y$, let $\mathscr{F}_{y}$ be the trace of the neighborhood filter $\mathscr{N}_{y}$ of the point $y$ in $Y$. Let $Z=\left\{\mathscr{F}_{y}: y \in Y\right\}$, and for $A \subseteq X$, let $0(A)$ denote $\left\{\mathscr{F}_{y}: A \in \mathscr{F}_{y}\right\}$. Then $\{0(U): U$ open in $X\}$ forms an open basis for a topology on $Z$ and $f: Y \rightarrow Z: y \rightarrow \mathscr{F}_{y}$ is a homeomorphism.

In this paper, we frequently identify the spaces $Y$ and $Z$ and for $A \subseteq X$, denote $\left\{y \in Y: A \in \mathscr{F}_{y}\right\}$ by $0(A)$. If $W$ is a set of open filters on a Hausdorff space $X$ and $W$ contains the set of neighborhood filters on $X$, then the topology on $W$ generated by $\{0(U)$ : $U$ open in $X\}$, where $0(U)=\{\mathscr{F} \in W: U \in \mathscr{F}\}$, is called the strict extension topology and $W$ with this topology is an extension of $X$. It is easy to verify the following properties for a regular extension $Y$ of $X$.

(4.4) If $V$ is regular open set in $Y$, then $0(V \cap X)=V$.

(4.5) If $V$ is an open set in $Y$, then $V \subseteq 0(V \cap X)$ and $\operatorname{cl}_{Y} V=$ $\operatorname{cl}_{Y}(V \cap X)=\operatorname{cl}_{Y}(0(V \cap X))$. 
A space $X$ is open combinatorially embedded in a space $Y$ if $Y$ is an extension of $X$ and for open subsets $U, V$ of $X$, then $\operatorname{cl}_{X} U \cap$ $\operatorname{cl}_{X} V=\varnothing$ implies $\operatorname{cl}_{Y} U \cap \operatorname{cl}_{Y} V=\varnothing$. The definition of "open combinatorially embedded" is based on those of "combinatorially embedded" [12] and "paracombinatorially embedded" [19]. A regular filter $\mathscr{F}$ on a space $X$ is a regular end if $\operatorname{cl} U \cap \operatorname{cl} V=\varnothing$ and $\mathscr{F}$ meets $U$ (i.e., $F \cap U \neq \varnothing$ for all $F \in \mathscr{F}$ ) imply $X \backslash \mathrm{cl} V \in \mathscr{F}$, where $U, V$ are open subsets of $X$. It is easy to verify that the neighborhood filter of a point in a regular space is a regular end and a regular end is a maximal regular filter. Let $w X$ denote the set of all regular ends on a space $X$. For each set $A \subseteq X$, let $0(A)$ denote $\{\mathscr{F} \in w X: A \in \mathscr{F}\}$. The set $\{0(U): U$ open in $X\}$ forms an open basis for a topology on $w X$.

Comments. In a normal (includes $T_{1}$ ) space, it is straightforward to show that:

(a) every regular filter is a completely regular filter,

(b) every maximal regular filter is a regular end and a maximal completely regular filter, and

(c) every maximal completely regular filter is a maximal regular filter.

Alexandroff [1] has characterized $\beta X$, the Stone-Čech compactification of a completely regular space $X$, as the set of all maximal completely regular filters on $X$ with the strict extension topology. Thus, for a normal space $X, w X=\beta X$, i.e., $w X$ and $\beta X$ are isomorphic as extensions of $X$. However, there is a completely regular space $X$ for which $w X \neq \beta X$. Consider the completely regular space $X_{2,2}$ described in Example 2.10. An argument similar to the argument used for the Tychonoff plank [13, p. 123] shows that $\beta X_{2,2}$ is the one-point compactification of $X_{2,2}$. So there is precisely one free maximal completely regular filter, denoted as $\mathscr{U}$, on $X_{2,2}$, namely, the trace on $X_{2,2}$ of the neighborhood filter of the point of infinity of $\beta X_{2,2}$. But $\mathscr{C}$ is not a regular end since the disjoint sets $T_{-1}$ and $T_{1}$ are closures of open sets and neither $X_{2,2} \mid T_{-1}$ nor $X_{2,2} \mid T_{1}$ belong to $\mathscr{C}$. By using the " $n$-corner" argument of [7], it is straightforward to show that $\mathscr{C}$ is the only free maximal regular filter on $X_{2,2}$. Since $\mathscr{C}$ is not a regular end, then $w X_{2,2}=X_{2,2}$ implying $w X_{2,2} \neq$ $\beta X_{2,2}$.

THEOREM 4.6. Let $X$ be a regular space.

(a) $w X$ is regular.

(b) $X$ is open combinatorially embedded in $w X$ by the function $e: X \rightarrow w X: x \rightarrow \mathscr{N}_{x}$.

(c) $w X$ is the injective maximum in the set of regular extensions in which $X$ is open combinatorially embedded. 
(d) A filter of $X$ is a regular end if and only if it is the trace of a neighborhood filter on $w X$.

\section{Proof.}

$A d(a)$. Since regular ends are maximal regular filters, then it easily follows that $w X$ is Hausdorff. Suppose $\mathscr{F}$ is a regular end and $\mathscr{F} \in O(U)$ for some open set $U \subseteq X$. Then $U \in \mathscr{F}$ and there are regular-open sets $V, B \in \mathscr{F}$ such that $\operatorname{cl}_{X} V \cong B \subseteq U$. Now $\mathscr{F} \in$ $O(V)$. Suppose $\mathscr{G} \in \mathrm{cl}_{w X}(O(V))$. Then $\mathscr{G}$ meets $V$, so $U \supseteq B=$ $X \mid \mathrm{cl}_{X}\left(X \backslash \mathrm{cl}_{X} B\right) \in \mathscr{G}$ implying $\operatorname{cl}_{w X}(O(V)) \subseteq O(U)$.

$A d(b)$. Since $X$ is Hausdorff, then $e$ is one-to-one. For an open set $U$ in $X, e^{-1}(O(U))=U$ and $e(U)=O(U) \cap\left\{\mathscr{N}_{x}: x \in X\right\}$. So, $e$ is an embedding function; $e(X)$ is dense in $w X$ since $O(U) \cap e(X)=$ $\left\{\mathscr{N}_{x}: x \in U\right\}$. Suppose $U$ and $V$ are open sets in $X$ such that $\operatorname{cl}_{X} U \cap$ $\operatorname{cl}_{X} V=\varnothing$. Let $\mathscr{F} \in \operatorname{cl}_{w X} U$. Then $\mathscr{F}$ meets $U$ implying $X \mid \mathrm{cl}_{X} V \in$ $\mathscr{F}$. So, $\mathscr{F} \notin \mathrm{cl}_{w X} V$ and $\mathrm{cl}_{w X} U \cap \mathrm{cl}_{w X} V=\varnothing$.

$A d(c)$. Let $Y$ be a regular space in which $X$ is open combinatorially embedded. Let $y \in Y$ and $\mathscr{F}_{y}$ denote $\left\{A \cap X: A \in \mathscr{N}_{y}\right\}$. Since $Y$ is regular, then $\mathscr{F}_{y}$ is a regular filter on $X$. To show $\mathscr{F}_{y}$ is a regular end on $X$, suppose $\operatorname{cl}_{X} U \cap \mathrm{cl}_{X} V=\varnothing$ where $U, V$ are open sets in $X$. Suppose $\mathscr{F}_{y}$ meets $U$. Then $y \in \mathrm{cl}_{Y} U$ implying $y \in Y \backslash \mathrm{cl}_{Y} V$. Hence, $X \backslash \mathrm{cl}_{X} V=\left(Y \backslash \mathrm{cl}_{Y} V\right) \cap X \in \mathscr{F}_{y}$. Using Theorem 4.3, it is straightforward to show that the function $Y \rightarrow w X: y \rightarrow \mathscr{F}_{y}$ is an embedding.

$A d(d)$. The proof follows immediately from the definition of the topology for $w X$.

A regular space $X$ is OCE-regular if $X$ has no proper regular extension in which $X$ is open combinatorially embedded. In particular, a regular-closed space is $O C E$-regular.

THEOREM 4.7. The following are equivalent for a regular space $X$.

(a) $X$ is OCE-regular.

(b) Every regular end converges.

(c) $X=w X$.

Proof. The proof is straightforward.

CoRollary 4.8. If $X$ is regular, then $w w X=w X$.

Our extension theory for the class of regular spaces is the class of $O C E$-regular spaces. We obtain a characterization of this extension 
theory by combining the methods of Harris [14] and Leader [20]. A relation $\delta$ on a set $X$ is called an $R$-proximity $[14,28]$ if $\delta$ satisfies:

(P1) $A \delta B$ implies $B \delta A$.

(P2) $\varnothing \varnothing A$ for all $A \subseteq X$.

(P3) $A \delta(B \cup C)$ if and only if $A \delta B$ or $A \delta C$.

(P4) $\{x\} \delta\{y\}$ if and only if $x=y$.

(P5) If $\{x\} \delta A$, then for some $B \subseteq X,\{x\} \delta X \backslash B$ and $B \phi A$.

For notational convenience, we will write $A<B$ for $A \phi X \backslash B, x \delta A$ for $\{x\} \delta A$, and $x<A$ for $\{x\}<A$. An $R$-proximity $\delta$ on a set $X$ induces a regular topology on $X$ defined in the usual way of $\operatorname{cl}_{X} A=$ $\{x \in X: x \delta A\}$. A filter $\mathscr{F}$ on $X$ is round if for each $F \in \mathscr{F}, G<F$ for some $G \in \mathscr{F}$, and a round filter $\mathscr{F}$ is a round end if, for open $U, V \subseteq X, \mathscr{F}$ meets $U$ and $\operatorname{cl}_{X} U \phi \mathrm{cl}_{X} V$ imply $X \backslash \mathrm{cl}_{X} V \in \mathscr{F}$. Some of the following facts are from Harris's article [14]; the rest are easily verified.

(4.9) [14] If $A \subseteq B<C \leqq D$, then $A<D$ and $X \backslash D<X \backslash A$.

(4.10) [14] If $A<B$ and $C<D$, then $A \cap C<B \cap D$ and $A \cup$ $C<B \cup D$.

(4.11) [14] If $A<B$, then $\operatorname{cl}_{X} A \subseteq B$ and $A \subseteq$ int $_{X} B$.

(4.12) A round end is a maximal round filter.

(4.13) The neighborhood filter of a point in $X$ is a round end.

(4.14) [14] Let $Y$ be a regular extension of $X$. The relation $\delta_{Y}$ on $X$ defined by $A \delta_{Y} B$ if $\mathrm{cl}_{Y} A \cap \mathrm{cl}_{Y} B \neq \varnothing$ is an $R$-proximity compatible with the topology of $X$ and a filter $\mathscr{F}$ on $X$ is round if and only if $\mathscr{F}$ is the trace of a regular filter on $Y$.

A c-proximity $\delta$ on $X$ is an $R$-proximity satisfying the property that if $\operatorname{cl}_{X} U \delta \mathrm{cl}_{X} V$ where $U$ and $V$ are open subsets of $X$, then some round end meets both $U$ and $V$.

LEMMA 4.15. Let $Y$ be a regular extension of $X$ and $\delta=\delta_{Y}$ the relation defined in 4.14 .

(a) If $U$ and $V$ are open subsets in $X$ and $U<V$, then $\operatorname{cl}_{Y}(0(U)) \subseteq$ $0(V)$.

(b) The trace on $X$ of a regular end on $Y$ is a round end; in particular, $\delta_{Y}$ is a c-proximity.

(c) $A$ round end on $X$ is the trace of a unique regular end on $Y$.

Proof.

$A d(a)$. Suppose $y \in \mathrm{cl}_{Y}(O(U))$. Since $U<V$ implies $\operatorname{cl}_{Y} U \cap$ $\operatorname{cl}_{Y}(X \backslash V)=\varnothing$ and since $\operatorname{cl}_{Y} U=\operatorname{cl}_{Y}(O(U))$, then $y \in Y \backslash \operatorname{cl}_{Y}(X \backslash V)$. Now $\left(Y \backslash \mathrm{cl}_{Y}(X \backslash V)\right) \cap X=V$, hence $y \in 0(V)$.

$A d(b)$. Let $\mathscr{C}$ be a regular end on $Y$ and $\mathscr{F}=\{G \cap X: G \in \mathscr{G}\}$. 
By $4.14, \mathscr{F}$ is a round filter. Suppose $\operatorname{cl}_{X} U \phi \mathrm{cl}_{X} V$ where $U, V$ are open subsets in $X$ and $\mathscr{F}$ meets $U$. Thus, $\mathscr{C}$ meets $O(U)$. Now $\mathrm{cl}_{X} U \grave{\phi \mathrm{cl}_{X}} V$ implies

$$
\varnothing=\operatorname{cl}_{Y} \operatorname{cl}_{X} U \cap \operatorname{cl}_{Y} \operatorname{cl}_{X} V=\operatorname{cl}_{Y} U \cap \operatorname{cl}_{Y} V=\operatorname{cl}_{Y} O(U) \cap \operatorname{cl}_{Y} O(V) .
$$

So, $Y \backslash \mathrm{cl}_{Y} O(V) \in \mathscr{C}$ implying $X \backslash \mathrm{cl}_{X} V=X \cap\left(Y \backslash \mathrm{cl}_{Y} O(V)\right) \in \mathscr{F}$.

$A d(c)$. Let $\mathscr{F}$ be a round end on $X$ and $\mathscr{C}$ the filter generated by the filter base $\{O(F): F \in \mathscr{F}\}$. It follows from (a) that $\mathscr{G}$ is a regular filter. Suppose $U$ and $V$ are open subsets of $Y$ such that $\operatorname{cl}_{Y} U \cap \operatorname{cl}_{Y} V=\varnothing$ and $\mathscr{G}$ meets $U$. Thus, $F \cap U \neq \varnothing$ for all $F \in$ $\mathscr{F}$. Now $\operatorname{cl}_{X}(U \cap X) \phi_{Y} \operatorname{cl}_{X}(V \cap X)$ implies $X \mid \mathrm{cl}_{X}(V \cap X) \in \mathscr{F}$; so, $O\left(X \mid \mathrm{cl}_{X}(V \cap X)\right) \in \mathscr{G}$. But $X \backslash \mathrm{cl}_{X}(V \cap X)=X \cap\left(Y \backslash \mathrm{cl}_{Y}(V \cap X)\right)=$ $X \cap\left(Y \backslash \mathrm{cl}_{Y} V\right)$, hence $Y \backslash \mathrm{cl}_{Y} V \in \mathscr{C}$, by 4.4. The uniqueness follows from the fact that a regular end is a maximal regular filter.

Let $\delta$ be an $R$-proximity on a set $X$. Let $c_{\delta} X=$ \{round ends on $X\}$, and for $A \subseteq X$, let $O(A)=\left\{\mathscr{F} \in c_{i} X: A \in \mathscr{F}\right\}$. The set $\{O(U): U$ open in $X$ \} forms an open basis for a topology on $c_{\delta} X$. $c_{\delta} X$ is a regular extension of $X$ (the proof is similar to the proof of Theorem 4.6). Define the relation $\Delta$ on $X$ by $A \Delta B$ if $\mathrm{cl}_{c} A \cap \mathrm{cl}_{c} B \neq \varnothing$ where $\operatorname{cl}_{c} A$ is the closure of $A$ in $c_{\hat{o}} X$. By Lemma $4.15 \Delta$ is a $c$-proximity on $X$, and by repeating the previous construction, we obtain the space $c_{\Delta} X$ which is denoted as $w_{\delta} X$.

THEOREM 4.16. Let $\delta$ be an R-proximity on $X$.

(a) $c_{\delta} X$ is a subspace of $w_{\hat{\delta}} X$.

(b) $w c_{i} X$ and $w_{\dot{o}} X$ are isomorphic extensions of $X$; in particular, $c_{\dot{\delta}} X$ is open combinatorially embedded in $w_{\dot{\delta}} X$.

(c) If $\delta$ is a c-proximity, then $c_{\hat{\delta}} X=w_{\hat{\delta}} X$.

Proof.

$A d(a)$. The neighborhood filter of a point in $c_{\delta} X$ is a regular end and its trace on $X$ is a 4 -round end by Lemma 4.15; hence, as sets $c_{\hat{o}} X \subseteq w_{\hat{o}} X$. Using Theorem 4.3, it is easy to check that the topology of $c_{\delta} X$ is the relative subspace topology of $w_{\delta} X$.

$A d(b)$. Since $w c_{\hat{o}} X$ and $w_{\hat{o}} X$ are regular extensions of $X$, then by Theorem 4.3, it suffices to show that the set of traces on $X$ of neighborhood filters on $w c_{\delta} X$ is the set of all $\Delta$-round ends, which follows from Lemma 4.15 (b, c) and Theorem 4.6 (d).

$A d(c)$. By (a) it suffices to show that a $\Delta$-round end $\mathscr{F}$ on $X$ is a $\delta$-round end. To show $\mathscr{F}$ is a $\delta$-round filter, let $F \in \mathscr{F}$. There are 
open sets $G$ and $H \in \mathscr{F}$ such that $\mathrm{cl}_{X} G<_{\Delta} \operatorname{int}_{X} \mathrm{cl}_{X} H\left(<_{\Delta}\right.$ is the $<$ corresponding to the proximity $\Delta)$ and $\operatorname{cl}_{X} H \subseteq F$. So, $\operatorname{cl}_{c} G \cap \operatorname{cl}_{c}\left(X \backslash \operatorname{cl}_{X} H\right)=$ $\varnothing$ implying $\operatorname{cl}_{X} G \phi \mathrm{cl}_{X}\left(X \mid \mathrm{cl}_{X} H\right)$ since $\delta$ is a $c$-proximity. This shows that $G<_{i} F$. To show $\mathscr{F}$ is a $\delta$-round end, suppose $\operatorname{cl}_{X} U \delta \mathrm{cl}_{X} V$ where $U$ and $V$ are open sets in $X$. Then $\operatorname{cl}_{c} \mathrm{cl}_{X} U \cap \mathrm{cl}_{c} \mathrm{cl}_{X} V=\varnothing$ implying $\operatorname{cl}_{X} U A \mathrm{cl}_{X} V$. If $\mathscr{F}$ meets $U$, then $X \backslash \mathrm{cl}_{X} V \in \mathscr{F}$ completing the proof that $\mathscr{F}$ is a $\delta$-round end.

COROLLARY 4.17. Let $Y$ be a regular extension of $X$. Let $\delta=$ $\delta_{Y}$ as defined in 4.14. Then $c_{\hat{o}} X=w_{\hat{o}} X$ and $w_{\hat{o}} X$ and $w Y$ are isomorphic extensions of $X$.

CoROllaRY 4.18. Let $X$ be a regular space, $\mathscr{R}(X)$ denote the set of $R$-proximities on $X$ compatible with the topology of $X$, and $\mathscr{E}(X)$ denote the set of OCE-regular extensions of $X$. Then the function $\Psi: \mathscr{R}(X) \rightarrow \mathscr{E}(X): \delta \rightarrow w_{i} X$ is onto.

If $\lambda$ is a relation on $X$ defined by $A \lambda B$ if $\operatorname{cl}_{w} A \cap \mathrm{cl}_{w} B \neq \varnothing$ where $\operatorname{cl}_{w} A$ is the closure of $A$ in $w_{o} X$, then using Theorem 4.16 (c) and the fact (by Lemma 4.15) that $\Delta$ is a $c$-proximity, it follows that $c_{\lambda} X=w_{\hat{o}} X$ and, hence, $\lambda=\Delta$. This shows that more than two iterations of the extension construction method yields nothing new. By Corollary 4.18, the set of $R$-proximities generate, via the function $\Psi$, all the $O C E$-regular extensions. By using Corollary 4.17 and Theorem 4.16, this result can be sharpened as follows:

Corollary 4.19. Let $X$ be a regular space and $\mathscr{R}_{c}(X)$ denote the set of c-proximities on $X$ compatible with the topology on $X$. Then $\Psi: \mathscr{R}_{c}(X) \rightarrow \mathscr{O}(X): \delta \rightarrow w_{i} X$ is onto.

We now characterize those $c$-proximities that induce the same $O C E$-regular extension.

Theorem 4.20. Let $X$ be a regular space. Suppose $\lambda, \delta \in \mathscr{R}_{c}(X)$. $A$ necessary and sufficient condition for $\Psi(\lambda)=\Psi(\delta)$ is that $\delta$ and $\lambda$ agree on regular closed sets, i.e., for every pair of open sets $U, V$ in $X, \mathrm{cl}_{X} U \delta \mathrm{cl}_{X} V$ if and only if $\mathrm{cl}_{X} U \lambda \mathrm{cl}_{X} V$.

Proof. Suppose $\Psi(\lambda)=\Psi(\delta)$, then a filter is a $\delta$-round end if and only if it is a $\lambda$-round end. Suppose $\operatorname{cl}_{X} U \delta \mathrm{cl}_{X} V$. Since $\delta$ is a $c$-proximity, there is a $\delta$-round end $\mathscr{F}$ (and hence a $\lambda$-round end) that meets both $U$ and $V$. It follows that $\operatorname{cl}_{X} U \lambda \mathrm{cl}_{X} V$. Similarly, $\mathrm{cl}_{X} U \lambda \mathrm{cl}_{X} V$ implies $\mathrm{cl}_{X} U \delta \mathrm{cl}_{X} V$. To show the converse, it suffices to prove that a $\delta$-round end $\mathscr{F}$ is a $\lambda$-round end. Let $F \in \mathscr{F}$. There are open sets $G, H \in \mathscr{F}$ such that $\operatorname{cl}_{X} G<_{i} \operatorname{int}_{X} \operatorname{cl}_{X} H$ and $\operatorname{cl}_{X} H \subseteq F$. 
So, $\mathrm{cl}_{X} G \phi \mathrm{cl}_{X}\left(X \mid \mathrm{cl}_{X} H\right)$ implying $\mathrm{cl}_{X} G \chi \mathrm{cl}_{X}\left(X \backslash \mathrm{cl}_{X} H\right)$. So, $\mathrm{cl}_{X} G<$, int $_{X} \mathrm{cl}_{X} H$ implying $G<_{\lambda} F$. To show $\mathscr{F}$ is a $\lambda$-round end, suppose $\mathrm{cl}_{X} U \chi \mathrm{cl}_{X} V$ and $\mathscr{F}$ meets $U$. Then $\operatorname{cl}_{X} U \phi \mathrm{cl}_{X} V$ implying $X \mid \mathrm{cl}_{X} V \epsilon$ $\mathscr{F}$. This completes the proof that $\mathscr{F}$ is a $\lambda$-round end.

For the $O C E$-regular extensions to be characterized, in contrast to simply being generated as done in Corollaries 4.18 and 4.19 , we must produce a subset of $\mathscr{R}_{c}(X)$ on which $\Psi$ is one-to-one and onto $\mathscr{E}(X)$. To accomplish this, we define an $R$-proximity $\delta$ on $X$ to be an OCER-proximity if for $A, B \subseteq X, A \delta B$ is equivalent to the existence of a round end meeting both $A$ and $B$. Certainly, an $O C E R$ proximity is a $c$-proximity. For a regular space $X$, let $\mathscr{R}_{0}(X)$ denote the set of $O C E R$-proximities on $X$ compatible with the topology on $X$.

Theorem 4.21. Let $X$ be a regular space. Then $\Psi: \mathscr{R}_{0}(X) \rightarrow$ $\mathscr{E}(X)$ is a bijection.

Proof. To show $\Psi$ is onto, let $Y \in \mathscr{E}(X)$ and $\delta=\delta_{Y}$. By Corollary 4.17, $Y=c_{\delta} X=w_{\delta} X$. It is straightforward to show that $\delta_{Y}$ is an OCER-proximity. To show $\Psi$ is one-to-one, suppose $\delta$ and $\lambda$ are $O C E R$-proximities such that $\Psi(\delta)=\Psi(\lambda)=Y$. Now $A \delta B$ if and only if $\mathrm{cl}_{Y} A \cap \mathrm{cl}_{Y} B \neq \varnothing$. Similarly, $\lambda=\delta_{Y}$. So, $\delta=\lambda$.

Let $\mathscr{E}_{r}(X)$ denote the set of regular extensions of a regular space $X$. Now, $\mathscr{E}_{r}(X)$ is partitioned by the equivalence relation $R$ defined by $Y R Z$ if $w Y$ and $w Z$ are isomorphic extensions of $X$. Each equivalence class of $\mathscr{E}_{r}(X)$ contains precisely one $O C E$-regular space which is characterized in the next theorem (the proof follows from Corollary 4.17 and Theorem 4.16).

THEOREM 4.22. Let $Y$ be an OCE-regular extension of a space $X$. Then $Y$ is the injective maximum among all the regular extensions $Z$ of $X$ such that $\delta_{Y}=\delta_{Z}$.

It is natural to inquire if $\mathscr{E}(X)$ for a regular space $X$ has a projective maximum- $w X$ seems to be a likely candidate. The following lemma and theorems answer this question.

Lemma 4.23. Let $X$ be a regular space and $Y, Z \in \mathscr{E}(X)$. If $Y$ is projectively larger than $Z$, then $\delta_{Y} \subseteq \delta_{Z}$.

Proof. There is a continuous function $f: Y \rightarrow Z$ which leaves $X$ pointwise fixed. Suppose $A, B \subseteq X$ and $A \phi_{Z} B$. Then $\mathrm{cl}_{Z} A \cap \mathrm{cl}_{Z} B=$ $\varnothing$. Since $f^{-1}\left(\mathrm{cl}_{Z} A\right) \supseteqq \operatorname{cl}_{Y} A$, then $\operatorname{cl}_{Y} A \cap \mathrm{cl}_{Y} B=\varnothing$ implying $A \phi_{Y} B$.

Recall that the Wallman proximity $\delta_{w}[14,11]$ on a regular space $X$ is defined by $A \delta_{w} B$ if $\operatorname{cl}_{X} A \cap \mathrm{cl}_{X} B \neq \varnothing . \delta_{w}$ is a $c$-proximity com- 
patible with the topology of $X$. Also, $w_{\hat{o}_{w}} X=w X$. Let $\delta_{0}=\delta_{w X}$. Now $\delta_{w} \subseteq \delta_{0}$, but $\delta_{w}=\delta_{0}$ is false as illustrated when $X$ is the deleted Tychonoff plank. Thus, in this case, $\delta_{w}$ is a $c$-proximity but not an $O C E R$-proximity. We now show that if $\mathscr{E}(X)$ has a projective maximum, it is $w X$.

Theorem 4.24. Let $X$ be a regular space. Then $w X$ is a projective maximal in $\mathscr{E}(X)$.

Proof. Let $Y \in \mathscr{E}(X)$ be projectively larger than $w X$. By Lemma 4.23, $\delta_{Y} \subseteq \delta_{0}$. However, $\delta_{w} \subseteq \delta_{Y}$. By Theorem 4.6, if $U$ and $V$ are open sets in $X$, then $\operatorname{cl}_{X} U \delta_{0} \mathrm{cl}_{X} U$ implies $\operatorname{cl}_{X} U \delta_{w} \mathrm{cl}_{X} V$. By Theorem 4.20, $\Psi\left(\delta_{0}\right)=\Psi\left(\delta_{Y}\right)$ and by Theorem 4.21, $Y$ and $w X$ are isomorphic extensions of $X$.

We now give a necessary condition for $w X$ to be a projective maximum in $\mathscr{E}(X)$. For a regular space $X$, Alexandroff [1] constructed an extension $\alpha X$ which is the set of all maximal regular filters on $X$ with the strict extension topology, i.e., the topology generated by the open basis $\{O(U): U$ open in $X\}$.

Theorem 4.25. If $X$ is a regular space and $w X$ is a projective maximum in $\mathscr{E}(X)$, then

(a) $w X$ and $\alpha X$ are isomorphic extensions of $X$,

(b) $w X$ is regular-closed, and

(c) if $U$ and $V$ are open sets in $X$ and $\operatorname{cl}_{X} U \cap \mathrm{cl}_{X} V=\varnothing$, then no maximal regular filter meets both $U$ and $V$.

Proof. Both (b) and (c) follow readily from (a). To prove (a), we need to show that a maximal regular filter $\mathscr{F}$ on $X$ is a regular end. Let $Y$ be the set $X \cup\{\mathscr{F}\}$ and define a set $U \subseteq Y$ to be open if $U \cap X$ is open in $X$ and $\mathscr{F} \in U$ implies $F \subseteq U$ for some $F \in \mathscr{F}$. $Y$ is a regular extension of $X$, and $w Y$ is an $O C E$-regular extension of $X$. There is a continuous function $f: w X \rightarrow w Y$ which leaves $X$ pointwise fixed. Thus, a regular end contains $\mathscr{F}$, and by $4.12, \mathscr{F}$ is a regular end.

An example of a locally compact, Hausdorff space $X$ in which there is no projective maximum in $\mathscr{E}(X)$ is the space $X_{2,2}$ used in the comment preceeding Theorem 4.6. So, $w X_{2,2}=X_{2,2}$ is not projectively larger than $\beta X_{2,2}$ which is an $O C E$-regular extension. This also shows that the mapping $X \rightarrow w X$ is not an epi-reflection [17] from the category of regular spaces and continuous functions to the full subcategory of $O C E$-regular spaces.

$\mathscr{E}(X)$, for a regular space $X$, contains all the regular-closed extensions of $X$. So, this extension theory subsumes the regular-closed 
extension theory by Harris [14]. In particular, an $R C$-proximity is an $O C E R$-proximity; even more, every maximal round filter relative to an $R C$-proximity is a round end. Also, a $W R$-proximity as defined by Harris [15] is a c-proximity.

Leader [20] introduced the term regulator. For our purposes a regulator [20, Cor. 2(a)] compatible with a regular space $X$ is a pair $(\mathscr{B},<)$ where $\mathscr{B}$ is a basis of regular-open sets of $X$ and " $<$ " is a binary relation on $\mathscr{B}$ satisfying

(1) $A<B \cong C$ implies $A<C$,

(2) $A<B$ implies $\mathrm{cl}_{X} A \subseteq B$, and

(3) for $x \in X, \mathscr{B}_{x}=\{B \in \mathscr{B}: x \in B\}$ is a round filter basis. For each regulator $(\mathscr{R},<)$ compatible with $X$, Leader constructs a regular extension (denoted as $c_{\infty} X$ ) which is the set of all $\mathscr{B}$-round ends (a $\mathscr{B}$-round end is a round filter $\mathscr{F}$ in $\mathscr{B}$ with the additional property that if $A<B$ where $A, B \in \mathscr{B}$ and $\mathscr{F}$ meets $A$, then $B \in \mathscr{F}$ ) with the strict extension topology.

Suppose $(\mathscr{B},<)$ is compatible with $X$. Define $A \delta B$ if $\mathrm{cl}_{\mathscr{B}} A \cap$ cl $_{B} B \neq \varnothing$ where $\mathrm{cl}_{\mathscr{S}} A$ denotes the closure of $A$ in $c_{\triangle} X$ (i.e., $A \delta B$ means some $\mathscr{B}$-round end meets both $A$ and $B$ ). By Lemma 4.15, $\delta$ is a $c$-proximity, and by Corollary 4.17, $X \subseteq c_{\delta} X \subseteq c_{\delta} X=w_{\delta} X$. On the other hand, if $\delta$ is an $R$-proximity on a set $X$ and $\mathscr{B}$ is a basis of regular-open sets, then the " $<$ " induced by $\delta$ and restricted to $\mathscr{B}$ is a regulator. So, there are three regular extensions $c_{\hat{\delta}} X, w_{\hat{o}} X$, and $c_{\mathscr{B}} X$ of $X$. If $\mathscr{B}^{\prime}$ is the set of all regular-open sets of $X$, then every $\mathscr{B}^{\prime}$-round end is a filter base for a $\delta$-round end and every $\delta$-round end is generated by some $\mathscr{B}^{\prime}$-round end; thus, $c_{\dot{\delta}} X$ and $c_{\mathscr{S},}, X$ are isomorphic extensions of $X$. In particular, if $\delta$ is a $c$-proximity, then $w_{\dot{o}} X$ and $c_{\mathscr{B}}, X$ are isomorphic extensions of $X$. Thus, the set of regular completions induced by regulators compatible with $X$ contains the set of $O C E$-regular extensions.

We express our thanks to the referee for valuable comments.

\section{REFERENCES}

1. P. Alexandroff, Bikompakte Erweiterung topologischer Räume, Mat. Sbornik N. S., 5 (1939), 420-429.

2. P. Alexandroff and P. Urysohn, Zur Theorie der topologischen Räume, Math. Ann., 92 (1924), 258-266.

3. B. Banaschewski, Über zwei Extremaleigenschaften topologischen Räume, Math. Nachr., 13 (1955), 141-150.

4. — Über Hausdorffsche-minimale Erweiterungen von Räumen, Arch. Math., 12 (1961), 355-365.

5. —_ Extensions of topological spaces, Canad. Math. Bull., 7 (1965), 1-22.

6. M. P. Berri, Minimal topological spaces, Trans. Amer. Math. Soc., 108 (1963), 97-105.

7. M. P. Berri and R. H. Sorgenfrey, Minimal regular spaces, Proc. Amer. Math. Soc., 14 (1963), 454-458. 
8. M. P. Berri, J. R. Porter and R. M. Stephenson, Jr., A survey of minimal topological spaces, General topology and its relations to modern analysis and algebra, Proc. Kanpur Top Conf. 1968, Acad. Press, New York, 1970, 93-114.

9. N. Bourbaki, Espaces minimaux et espaces complètement séparés, C. R. Acad. Sci. Paris, 212 (1941), 215-218.

10. - General Topology, Part 1, Addision-Wesley, Reading, Mass., 1966.

11. E. Čech, Topological Spaces, Interscience, New York, 1966.

12. E. Čech and J. Novák, On regular and combinatorial embedding, Časopis Pěst. Mat., 72 (1947), 7-16.

13. L. Gillman and M. Jerison, Rings of Continuous Functions, Van Nostrand, New York, 1960.

14. D. Harris, Regular-closed spaces and proximities, Pacific J. Math., 34 (1970), 675686.

15. - Regular-closed spaces and structures, submitted.

16. H. Herrlich, $T_{\nu}$-Abgeschlossenheit und $T_{\nu}$-Minimalität, Math. Z., 88 (1965), 285294.

17. - Categorical topology, General Topology and its Applications, 1 (1971), 1-15. 18. M. Katětov, Über H-abgeschlossene und bikompakt Räume, Časopis Pěst. Mat., 69 (1940), 36-49.

19. - On H-closed extensions of topological spaces, ibid., 72 (1947), 17-32.

20. S. Leader, Regulated bases and completions of regular spaces, Fund. Math., 68 (1970), 279-287.

21. J. Mioduszewski, Remarks on Baire Theorem for H-closed spaces, Coll. Math., 23 (1971), 39-41.

22. J. R. Porter, Minimal R(wo) spaces, Notices Amer. Math. Soc., 16 (1969), 218.

23. A. Ramanathan, A characterization of maximal-Hausdorff spaces, J. Indian Math. Soc. N. S., 11 (1947), 73-80.

24. C. T. Scarborough, Minimal Urysohn spaces, Pacific J. Math., 27 (1968), 611-617.

25. R. M. Stephenson, Jr., P-minimal and P-closed spaces, Ph. D. dissertation, Tulane University, 1967.

26. - A countable minimal Urysohn space is compact, Proc. Amer. Math. Soc., 22 (1969), 625-626.

27. - Spaces for which the Stone-Weierstrass theorem holds, Trans. Amer. Math. Soc., 133 (1968) 537-546.

28. D. V. Thampuran, Regular spaces and relations, Mat. Vesnik, 7 (22) (1970), 395400.

29. P. Urysohn, Über die Machtigkeit der zusammenhängenden Mengen, Math. Ann., 94 (1925), 262-295.

30. G. A. Viglino, $\bar{T}_{n}$ spaces, Notices Amer. Math. Soc., 16 (1969), 846.

Received October 29, 1971 and in revised form April 7, 1972. The research of the first author was partially supported by a University of Kansas research grant no. 34165038 and of the second author by a National Science Foundation Traineeship.

The University of Kansas

AND

Fort Hays Kansas State College 



\section{PACIFIC JOURNAL OF MATHEMATICS}

EDITORS

\author{
H. SAMELSON \\ Stanford University \\ Stanford, California 94305 \\ C. R. HOBBY \\ University of Washington \\ Seattle, Washington 98105
}

\author{
J. DUGUNDJI \\ Department of Mathematics \\ University of Southern California \\ Los Angeles, California 90007 \\ RICHARD ARENS \\ University of California \\ Los Angeles, California 90024
}

\section{ASSOCIATE EDITORS}

E. F. BECKENBACH

B. H. NeUManN

F. WOLF

K. YosHIDA

\section{SUPPORTING INSTITUTIONS}

UNIVERSITY OF BRITISH COLUMBIA

CALIFORNIA INSTITUTE OF TECHNOLOGY

UNIVERSITY OF CALIFORNIA

MONTANA STATE UNIVERSITY

UNIVERSITY OF NEVADA

NEW MEXICO STATE UNIVERSITY

OREGON STATE UNIVERSITY

UNIVERSITY OF OREGON

OSAKA UNIVERSITY
UNIVERSITY OF SOUTHERN CALIFORNIA

STANFORD UNIVERSITY

UNIVERSITY OF TOKYO

UNIVERSITY OF UTAH

WASHINGTON STATE UNIVERSITY UNIVERSITY OF WASHINGTON

AMERICAN MATHEMATICAL SOCIETY NAVAL WEAPONS CENTER 


\section{Pacific Journal of Mathematics}

\section{Vol. 45, No. $1 \quad$ September, 1973}

William George Bade, Complementation problems for the Baire classes .......... 1

Ian Douglas Brown, Representation of finitely generated nilpotent groups ........ 13

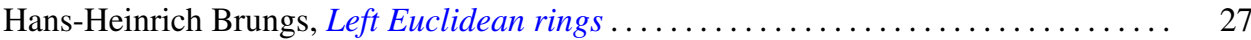

Victor P. Camillo and John Cozzens, A theorem on Noetherian hereditary rings ..... 35

James Cecil Cantrell, Codimension one embeddings of manifolds with locally flat

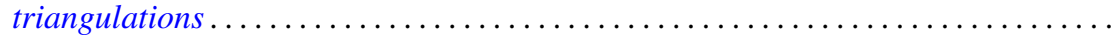

L. Carlitz, Enumeration of up-down permutations by number of rises . . . . . . . . . .

Thomas Ashland Chapman, Surgery and handle straightening in Hilbert cube

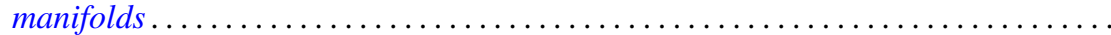

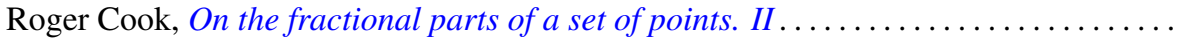

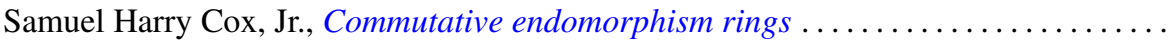

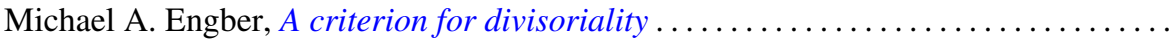

Carl Clifton Faith, When are proper cyclics injective . . . . . . . . . . . . . . 97

David Finkel, Local control and factorization of the focal subgroup . . . . . . . . . 113

Theodore William Gamelin and John Brady Garnett, Bounded approximation by

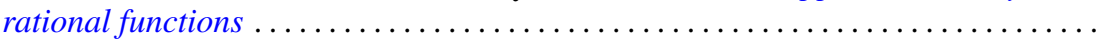

Kazimierz Goebel, On the minimal displacement of points under Lipschitzian

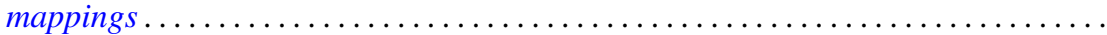

Frederick Paul Greenleaf and Martin Allen Moskowitz, Cyclic vectors for representations associated with positive definite measures: nonseparable

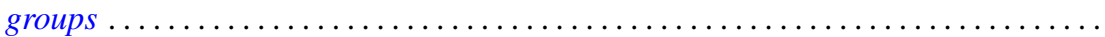

Thomas Guy Hallam and Nelson Onuchic, Asymptotic relations between perturbed linear systems of ordinary differential equations .

David Kent Harrison and Hoyt D. Warner, Infinite primes of fields and completions. .

James Michael Hornell, Divisorial complete intersections . ......

Jan W. Jaworowski, Equivariant extensions of maps ..............

John Jobe, Dendrites, dimension, and the inverse arc function .. .

Gerald William Johnson and David Lee Skoug, Feynman integrals of non-factorable

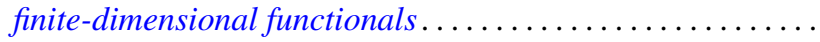

Dong S. Kim, A boundary for the algebras of bounded holomorphic functions ...... 269

Abel Klein, Renormalized products of the generalized free field and its derivatives ... 275

Joseph Michael Lambert, Simultaneous approximation and interpolation in $L_{1}$ and

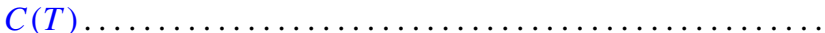

Kelly Denis McKennon, Multipliers of type $(p, p)$ and multipliers of the group

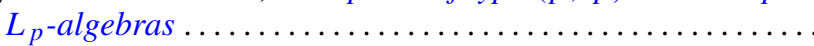

William Charles Nemitz and Thomas Paul Whaley, Varieties of implicative

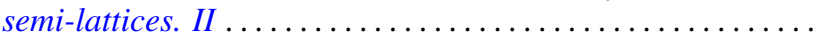

Donald Steven Passman, Some isolated subsets of infinite solvable

Norma Mary Piacun and Li Pi Su, Wallman compactifications on E-completely

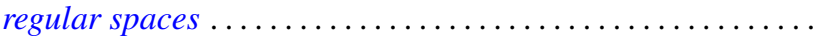

Jack Ray Porter and Charles I. Votaw, $S(\alpha)$ spaces and regular Hausdorff extensions....

Gary Sampson, Two-sided $L_{p}$ estimates of convolution transforms .

Ralph Edwin Showalter, Equations with operators forming a rig
Raymond Earl Smithson, Fixed points in partially ordered sets .

Victor Snaith and John James Ucci, Three remarks on symmetric products and

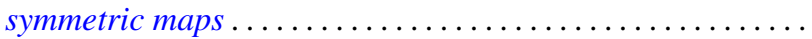

